\title{
ROLE OF ENVIRONMENTAL FACTORS ON THE REPRODUCIBILITY OF LEMNA TEST
}

\author{
I. Kiss, * NóRa Kováts and Tímea Szalay \\ Institute of Biology, University of Veszprém, Veszprém, Hungary
}

(Received: March 14, 2000; accepted: June 2, 2000)

\begin{abstract}
Lemna minor is a species easy to collect and culture in laboratory, and can give rapid test results. However, in order to standardise toxicity tests using Lemna minor as test organism, it is important to find out what natural variability different populations might have. Five Lemna populations were used for comparison. It contained two standard cultures and three populations collected in natural habitats. Potassium dichromate was applied as test material. Lemna populations cultured under the same conditions showed different $T_{D}$ and $\mathrm{LC}_{50}$ values. There is an inverse relation between the sensitivity and $T_{D}$ of the strains. It is supposed that growth rate and sensitivity of Lemna populations depend on environmental factors characterising the habitat in which the given popluation originally lives.
\end{abstract}

Keywords: Bioindication - Lemna minor - toxicity test

\section{INTRODUCTION}

In ecotoxicological testing, biomarkers have an increasing significance $[3,4]$. The basis of bioindication is that any living organism can show normal functions only under well-determined physical-chemical conditions. Some species are tolerant to pollution in a wide range, while others are specifically sensitive to changes in environmental factors. The significance of bioindication is the possibility of an easy estimation of the environmental toxicity at low (sublethal) doses.

Duckweed (Lemna minor) is a relatively new bioindicator suggested by EPA (1996) and OECD experts [1, 2,3]. The objective of this toxicity test is to quantify substance-related effects on vegetative growth. The main advantages: L. minor is a species easy to collect and culture in laboratory, and can give rapid test results [7]. However, a validation procedure is required before the final standardization. For this reason an OECD international ring test was intitiated in which the Biological Institute of the University of Veszprém was invited to participate.

Presently it has been suggested that some genetic variability in the response to toxins can occur with Lemna, however there is currently insufficient data on this source

*Corresponding author; E-mail: zoologia@almos.vein.hu 
of variability. An important question may be: what natural variability different $L$. minor populations might have and is there a specific clone for use with the OECD toxicity test guideline?

In order to assess the extent of the variability in response to toxic pollution, tests were carried out with $L$. minor populations collected at different habitats under standard conditions, using potassium dichromate as toxic agent.

Potassium dichromate was applied as test material. Chromium exists in aquatic systems in two oxidation states (chromium VI and chromium III). While chromium III has a low toxicity to aquatic life, and is generally regulated based on the human health hazard associated with domestic water supplies, chromium VI is highly toxic to some forms of aquatic life. It has been suggested that the $\mathrm{Cr}(\mathrm{VI})$ ion can be used as a general reference toxicant in river water, lake water or wastewater samples. The concentration-effect relationships, however, are different, depending on the test organisms. [7] The thermodynamically stable species of $\mathrm{Cr}$ is Cr VI. There are also a number of reactions that tend to convert $\mathrm{Cr}$ VI to $\mathrm{Cr}$ III in toxic conditions, including photoreduction.

\section{MATERIALS AND METHODS}

\section{Lemna populations}

Five Lemna populations were used for comparison. Amongst them there were two standard cultures (L1 and L2), L3 was collected in a natural habitat in the area of the Kis Balaton Water Protection System which is very poor in nutrients and oxygen, L4 from a channel in Keszthely which is rich in nutrients and well-oxygenated and the fifth sample, L5 was collected in a small pond which collects the leachate of a communal waste disposal site, near Herend.

\section{Test conditions (Static test)}

Controls and test vessels were kept in an incubator illuminated by continuous warm fluorescent lighting. Light intensity was $8000( \pm 100)$ lux as measured at the surface of the test solution. Temperature in the test vessel was $23 \pm 2{ }^{\circ} \mathrm{C}$. For Lemna minor SIS [5] growth medium was prepared and sterilised according to the OECD guideline. Erlenmeyer flasks of $150 \mathrm{ml}$ volume were used as control and test vessels, with a randomised location.

\section{Experimental procedure}

One control culture and four concentrations of potassium dichromate were applied in case of Lemna 1 and Lemna 5, while five concentrations of the test substance were 
applied in case of Lemna 2-4. The test concentrations were prepared by dilution of a stock solution having been prepared by dissolving the test substance in the test medium.

The test was terminated 7 days after the plants were inoculated into the test vessels. Frond numbers and the appearance of the colonies were recorded at the beginning, then at $72 \mathrm{~h}, 120 \mathrm{~h}$ and at the termination of the study.

Changes in the biomass were also detected at the same occasions as the frond numbers. The assessment of the biomass was based on the fresh weight.

\section{Statistical evaluation}

During the experiment the data of each replicate were collected individually. Toxicity evaluation was based on the average values of $\mu$ per each replicate. These slopes were calculated by the regression analysis in Excel. Five replicates were employed. For comparison of replicates we used the SPSS statistical program package, first ANOVA, than Duncan test (oneway) for multiple comparison of dose groups and evaluating the significance level of the effects.

The value of $\mathrm{LC}_{50}$ has been calculated on the basis of $\% \mathrm{I}_{\mathrm{r}}$ with linear regression (following log transformation of concentration data), and graphical method. For this analysis INSTAT computer program was used: for an evaluation of regression lines, their confidence limits and deviation from the linearity. The confidence interval of $\mathrm{LC}_{50}$ was also determined.

\section{Analysis of the data}

From the natural logarithm of fronds $-\ln (F)$ specific growth rate $(\mu)$ was calculated. From the same curve we can derive the doubling time $\left(T_{D}\right)=\ln 2 / \mu$.

Specific growth rate $(\mu)$ was calculated as follows:

where:

$$
\mu=\left\{\ln \left(N_{t}\right)-\ln \left(N_{0}\right)\right\} / t
$$

- $N_{0}$ is the frond number at time zero (at the beginning of the test)

- $N_{t}$ is the frond number in the vessel after time $t$

- $t$ is the time interval.

Percentage average growth rate (\%Ir) was calculated as follows:

where:

$$
\% \operatorname{Ir}=(\mathrm{C} \mu-\mathrm{T} \mu) * 100 / \mathrm{C} \mu
$$

- $\mathrm{C} \mu$ is the growth rate in the control

- $\mathrm{T} \mu$ is the growth rate in the test group 
Multiple comparison of dose groups and evaluating the significance level of the effects was made by ANOVA, then Duncan test.

Basic table (Table 1) for calculating average specific growth rate and percentage inhibition of the growth rate is demonstrated below.

Table 1

\begin{tabular}{|c|c|c|c|c|}
\hline \multicolumn{5}{|c|}{ Average specific growth rate $(\mu)$} \\
\hline Control & $2.48 \mathrm{mg} / \mathrm{l}$ & $4.96 \mathrm{mg} / \mathrm{l}$ & $9.91 \mathrm{mg} / 1$ & $19.82 \mathrm{mg} / \mathrm{l}$ \\
\hline 0.227 & 0.187 & 0.105 & 0.138 & 0.127 \\
\hline 0.235 & 0.139 & 0.047 & 0.096 & 0.065 \\
\hline 0.288 & 0.198 & 0.143 & 0.124 & 0.065 \\
\hline 0.182 & 0.164 & 0.100 & 0.071 & -0.026 \\
\hline 0.186 & 0.088 & nd & 0.115 & nd \\
\hline Average \pm SD & & & & \\
\hline $0.224 \pm 0.043$ & $0.155 \pm 0.044$ & $0.099 \pm 0.039$ & $0.109 \pm 0.026$ & $0.064 \pm 0.052$ \\
\hline \multicolumn{5}{|c|}{ Percentage inhibition of the growth rate (\%Ir) } \\
\hline & 17.62 & 53.74 & 39.65 & 44.05 \\
\hline & 40.85 & 80.00 & 59.15 & 72.34 \\
\hline & 31.25 & 50.35 & 56.94 & 77.43 \\
\hline & 9.90 & 45.05 & 60.99 & 114.29 \\
\hline & 44.62 & nd & 38.17 & nd \\
\hline Average \pm SD & $29.83 \pm 18.05$ & $46.91 \pm 23.19$ & $56.86 \pm 11.93$ & $84.32 \pm 25.21$ \\
\hline
\end{tabular}

\section{RESULTS}

\section{Lemna 1}

Concentration of potassium dichromate (as Cr) was: $2.48-4.87$ - $9.91-19.82 \mathrm{mg} / \mathrm{l}$. We used also a high cocentration, as much as $39.64 \mathrm{mg} / \mathrm{l}$, however, it killed the test organism completely, therefore it was discarded.

The value of $\mathrm{LC}_{50}$ was calculated on the basis of $\% \mathrm{I}_{\mathrm{r}}$ with linear regression.

$\% \mathrm{I}_{\mathrm{r}}=$ percentage inhibition of specific growth rate (Fig. 1).

$\mathrm{LC}_{50}=6.096(2.851-9.334) \mathrm{mg} / \mathrm{l}$. 


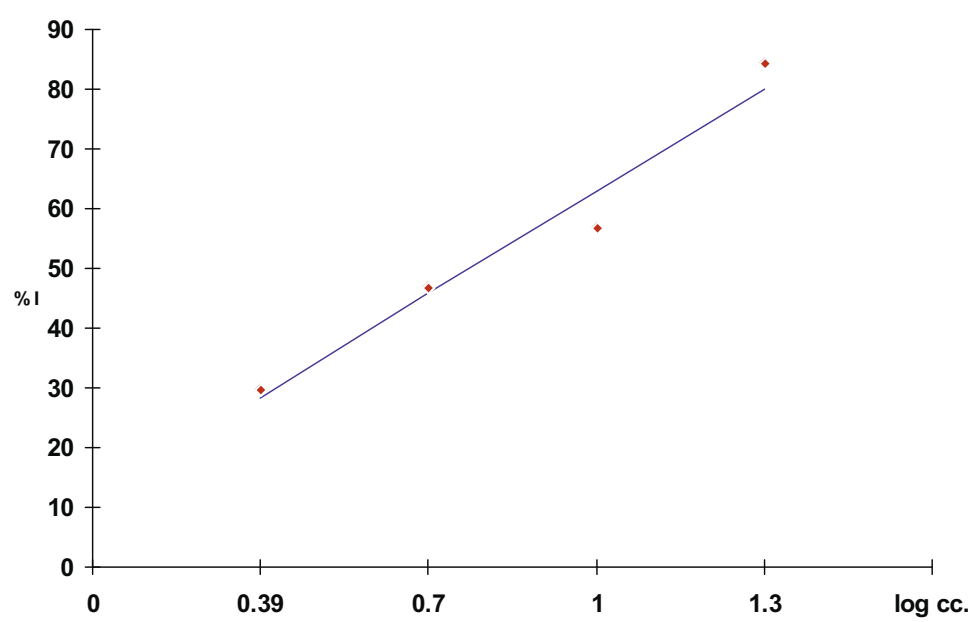

Fig. 1. Percentage inhibition (\%I) of specific growth rate of Lemna 1 by $\mathrm{K}_{2} \mathrm{Cr}_{2} \mathrm{O}_{7}$

$\mathrm{T}_{\mathrm{D}}=3.0$ days and the growth in the control was exponential.

\section{Lemna 2}

The procedure was the same as above. The only difference: a wider concentration range was used as below:

$1.24-2.48-4.96-9.91-19.82 \mathrm{mg} / 1$

$\mathrm{LC}_{50}$ (based on \% inhibition) $=2.661(1.758-3.550) \mathrm{mg} / 1$

$\mathrm{T}_{\mathrm{D}}=4.5$ days and the growth in the control was exponential.

\section{Lemna 3}

Concentrations used were the same as above.

$\mathrm{LC}_{50}=3.81(1.91-5.0) \mathrm{mg} / 1$

$\mathrm{T}_{\mathrm{D}}=3.46$ day

\section{Lemna 4}

Concentrations used were the same as above.

$\mathrm{LC}_{50}=2.63(1.90-3.0) \mathrm{mg} / 1$

$\mathrm{T}_{\mathrm{D}}=5.1$ day 


\section{Lemna 5}

Concentrations used were: $2.48-4.96-9.91-19.82 \mathrm{mg} / \mathrm{l}$

$\mathrm{LC}_{50}=10.0(9.5-11.0) \mathrm{mg} / \mathrm{l}$

$\mathrm{T}_{\mathrm{D}}=2.878$ day

\section{DISCUSSION AND CONCLUSIONS}

$\mathrm{T}_{\mathrm{D}}$ and $\mathrm{LC}_{50}$ values of the different Lemna cultures are summarised in Fig. 2. It seems that there is an inverse relation between the sensitivity and $T_{D}$ of the strains: the more the $\mathrm{T}_{\mathrm{D}}$ is, the less the $\mathrm{LC}_{50}$ value is: the sensitivity to toxic effect increased.

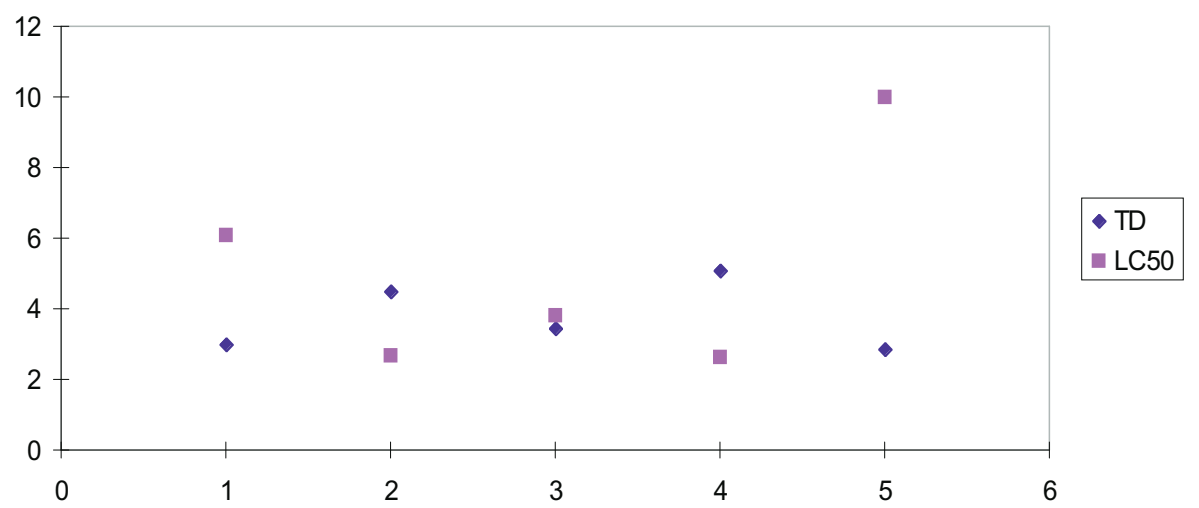

Fig. 2. $\mathrm{T}_{\mathrm{D}}$ and $\mathrm{LC}_{50}$ values of $\mathrm{L} 1-\mathrm{L} 5$ populations

As literature and relevant standards cite, Lemna minor is suitable to test the toxicity of surface waters. Assessment of such effects was one of the purposes of the OECD ring test. Our tests verify the sensitivity of the duckweed for such purpose, however, standardisation of laboratory tests may cause some problem, especially with regard of the origin of Lemna cultures.

Even Lemna populations cultured under the same, standard conditions might show different $T_{D}$ values which in turn determine the sensitivity of the organisms. It is also confirmed by other authors (Whitehouse, personal com.)

Any comparison between toxicity of various substances at laboratory level can only be made when $T_{D}$ is strictly adjusted to a constant value. Our study also prove that this is an essential term of standardisation of Lemna toxicity tests.

It might be supposed that growth rate and therefore sensitivity of Lemna populations depend on environmental factors characterising the habitat in which the given population originally lives. We might suppose that nutrient availability are among 
these factors, as the example of L3-L4 shows. Also, populations living in already polluted environment (leachate of the waste disposal site, L5) might develop a certain resistance to toxic chemicals, which is demonstrated by the extremely high $\mathrm{LC}_{50}$ value, $10 \mathrm{mg} / \mathrm{l}$.

The next step in this study will be to reveal which are the crucial factors determining the sensitivity of Lemna and perhaps a semi-quantitative relationship might be found between the given environmental variable(s) and the sensitivity. In order to establish such relationship, other toxic materials than potassium dichromate will be tested as well.

In case our further research prove that even in the case of different toxic materials environmental variables show strong correlation with the sensitivity of Lemna populations, such natural populations might be used for the habitat-specific indication of the toxic pollution occurring at the given habitat.

\section{REFERENCES}

1. Huebert, D. B., Shay, J. M. (1993) Considerations in the assessment of toxicity using duckweeds. Environmental Toxicol. and Chem. 12, 481-483.

2. Jenner, H. A., Jansen-Mommen, J. P. M. (1989) Phytomonitoring of pulverized fuel ash leachates by the duckweed Lemna minor. Hydrobiologia, 188/189, 361-366.

3. Lopez-Barera, J. (1995) Biomarkers in Ecotoxicology. Eurotox Proc. Toxicology in transition. Springer V. Berlin, 57-79.

4. Moriarty, F. (1990) Ecotoxicology. Acad. Press, San Diego.

5. Swedish Standard Institute (1995) Water quality - Determination of growth inhibition (7-d) Lemna minor, duckweed. SS 0282 13. 1995, 15 p.

6. United States Environmental Protection Agency (1996) OPPTS 850.4400 Aquatic Plant Toxicity Test using Lemna spp. Public draft, EPA 712-C-96-156. 8 pp.

7. Wang, W. (1990) Literature review on duckweed toxicity testing. Environmental Res. 52, 7-22. 
